\title{
The Photometric Stereo Approach and the Visualization of 3D Face Reconstruction
}

\author{
Muhammad Sajid Khan ${ }^{1}$, Zabeeh Ullah ${ }^{2}$, Maria Shahid Butt ${ }^{3}$, Zohaib Arshad ${ }^{4}$, Sobia Yousaf ${ }^{5}$ \\ College of Computer Science, Sichuan University, Sichuan Chengdu China ${ }^{1}$ \\ Federation University Australia ${ }^{2}$ \\ University Institute of Information Technology, PMAS Arid, Agriculture University Rawalpindi, Pakistan ${ }^{3}$ \\ Army Public College of Management \& Sciences, Rawalpindi Punjab Pakistan ${ }^{4,5}$
}

\begin{abstract}
The 3D Morphable models of the human face have prepared myriad of applications in computer vision, human computer interaction and security surveillances. However, due to the variation in size, complexity of training data set, the landmark mapping, the representation in real time and rendering or synthesis of images in three dimensional is limited. In this paper, we extend the approach of the photometric stereo and provide the human face reconstruction in three dimensional. The proposed method consists of two steps. First it automatically detects the face and segment the iris along with statistical features of pupil location in it. Secondly it provides the selection of minimum six features and where iris process to generate the 3D face. In compare with existing methods our approach provides the automation which produces more better and efficient results in contrast to the manual methods.
\end{abstract}

Keywords-3D face; photometric stereo; reconstruction; recognition; feature selection

\section{INTRODUCTION}

The significance of face applications grows rapidly, face recognition methods [1] using three-dimensional data has been getting more attention in the area of computer vision because of two-dimensional face recognition problems such as pose and illumination. The construction of the human face in $3 \mathrm{D}$ is a difficult task in the area of pattern recognition and graphic design. In the last few years, different methods have been stated successful for recognition such as identification for authentication. Though few algorithms have worked well in speed and accuracy, improvements are still required. The basic reconstruction and final modeling were two steps for faces reconstruction. 3D face reconstructions have different domains such as recognition, synthesis, features detection, resolution and image matching. Recognition uses identity data set [2] as input for intra purpose variation. Synthesis takes pie data set for producing Multi-model face recognition. Feature detection uses raw images and processed these images in real time. Resolution utilizes quantized depth map that gives the superresolution for color. Image matching uses feature pairs that enhance the accuracy of models. Face image processing is generally used in different real-life applications such as machine interaction by robotics and human, cosmetic surgery and security applications. 3D reconstruction is the procedure of capturing the appearance and shape of existent objects. The process may be accomplished by active and passive methods. The classification of $3 \mathrm{D}$ face terms is based on features and reconstruction. Active methods related with a reconstruction of mechanical objects and radiometric like laser, ultrasound and visible light. Passive methods involve the radiation of light in 3D construction and measurement of emission such as image sensors. Accumulating the 3D particulars about the object is known as the data acquisition process, which is an essential part of the reconstruction method and plays a vital role in computer vision applications. To complete the reconstruction, a process is required to fit and makeover a generic face after precise data acquisition. It is fundamental to produce realistic human face models in human face reconstruction. The reconstruction process needs a transformation from two dimensional to three-dimensional spaces. A 3D face is used in various applications [3] such as animation, video meeting, face recognition, games and facial synthesis. It is challenging to control the acute problems and to improve the development systems of presenting three dimensional images. Using 2D response efforts to generate an advanced output of 3D images numerous developments of 3D frameworks have been achieved. Face reconstruction models are briefly explained in this paper, and we focus on different indirect $3 \mathrm{D}$ reconstruction methods. In remaining part of this paper, we primarily review the indirect 3D reconstruction methods and its importance, focusing on recent growths. Face reconstruction is an extensive subject; numerous topics are covered in different fields like face recognition, detection, texture and alignment. In Section 3 we briefly discuss the proposed photometric stereo approach and its image based method. A 3D reconstruction can be done through two methods; direct and indirect method. The 3D point is pursued in a way to reduce the reconstruction error between the measured similarities and the solution is known as direct method.

\section{LITERATURE REVIEW}

First, Photometric stereo methods are used for the computation of surface reconstruction. It took single images under distinctive light conditions from the same focusing point. This technique solved the inappropriate posture of the image under various illumination states. Though every image has its own unique reflectance map and each point is reliant on and provides a specific set of location. It's simple, easy to implement and have low computational cost uses extra lights only. Photometric stereo partitioned into the traditional and general stereo. The traditional photometric stereo camera based on linear radiometric response functions and orthographic prediction. Lambert's reflectance model [4] appropriate on surface reflectance and similar light direction for all sight 
points. The shadow of an object is ignored in traditional photometric stereo [6] whereas the general photometric stereo does not strict to follow the norms and suffering from the problems of imprecise lighting. Photometry is the technological know-how of the size of the light, in terms of its supposed brightness to the human eye. Stereopsis is a term that is most usually used to consult the notion of intensity and three-dimensional structure acquired at the bases of visual records originating from two eyes through people with usually advanced binocular vision. Photometric stereo is a method for estimating the surface normal of objects by perceiving it under different light conditions. Given enough light sources from different angles, the depth information can be recovered. The concept of photometric stereo is quite simple. Photometric stereo methods use fixed cameras and light directions. The limitation of this model is varying from resources to resources. Uncalibrated model is usually used as the Lambertian model. With uncalibrated model use the calibrated lighting. The Deep Photometric stereo acquires from the different measurements of the vector and surface normal. It involves seven layers; six compact layers and one shadow layer. If there is no shadow then it will increase the accuracy of the proposed method [7]. Noise and outliers must be reduced to get the most accurate photometric stereo results. Shadow reflections are seen as outliers. Depth, albedo, and lighting combined to improve accuracy performance. It is computationally very expensive but doesn't have the denominator issues. Calibrated and noncalibrated methods [8] are used. Mean estimator is used for handling the difficulties in the shadow reflection of an image. Shape from shading is the most challenging task in photometric stereo. A semi calibrated photometric stereo [9] know the position but the intensity of the light is unknown. It resolved the albedo and light reflection problems. It calculates the depth and then shadows reflectance map estimation. It is robust and gives the best results. J Roth et al. [10], proposed a photometric stereo-based approach in which 2D landmarks and basic template are given to the iterative process that reconstruct the surface by estimating the 3D landmarks and photometric normal. Sun Y et al. [11][12], uncalibrated Photometric Stereo approach used, reconstructs 3D face by iteratively estimates the different illumination conditions with the help of face albedo and surface normal.

\section{PROPOSED METHOD}

In this paper, the proposed system used to reconstruct the $3 \mathrm{D}$ faces using the Photometric Stereo approach and shows the visualization of $3 \mathrm{D}$ reconstruction. A human face is close enough in standard conditions to be a good estimation. Our proposed method determines the light direction from the eye. In proposed method its combine the normal vectors process and the Frankot-Chellappa algorithm. As it is a name of twodimensional integration but in this case, using for three dimensional. In order to compute the surface gradients of the selected image and take a Fourier transform to get the surface image. Before applying the Frankot-Chellappa algorithm using eye center, eye radius and highlight features from eye image and these feature process to provide a $3 \mathrm{D}$ reconstruction.

\section{A. Proposed Algorithm}

1. Start

2. Load the set of images(under different lighting condition)

3. Find rectangular region using mouse selection from the input image (I).

4. Compute the mask image;

a. Select rectangle of face from the sequence of images.

b. Select points from the selected region (minimum 6 points).

c. Binary mask is created.

d. Perform whole filling on the mask obtained in previous step.

5. Gets the iris image (both left and right image) using rectangular mouse selection

6. Get eye locations from the sequence of images

7. Compute features (eye center, radius, highlight, iris image, eye image) from eye image

8. Create surface normal of the gradient map

9. Construction of surface image using FrankotChellappa Algorithm

a. Compute the surface gradient of mask and normal image

b. Take Fourier transform

c. Integrate in the frequency domain by phase shifting by $\mathrm{pi} / 2$ and weighting the Fourier coefficients by their frequencies in $\mathrm{x}$ and $\mathrm{y}$ and then dividing by the squared frequency. eps is added to the denominator to avoid division by 0 .

d. Get the surface image

10. End

\section{B. Frame Work of Proposed Method}

Human face follows the Lambertian reflectance property.

$\mathrm{V}=\mathrm{m}(\mathrm{s} . \mathrm{D})$

In equation (1) $\mathrm{V}$ is a known vector of observed intensities, $\mathrm{s}$ is the unknown surface normal and $\mathrm{D}$ is a light source direction.

$\mathrm{D}-1 \mathrm{~V}=\mathrm{ms}$

$\mathrm{DTV}=\mathrm{DT}$ m (s. D)

(DT D)-1 DTV = ms

In equation (2) ms are the surface normal that are to be computed. Normal vector have length Inverse can be obtained by simply multiplying DT on both sides. Overview of our proposed algorithm is shown in Fig. 1. 


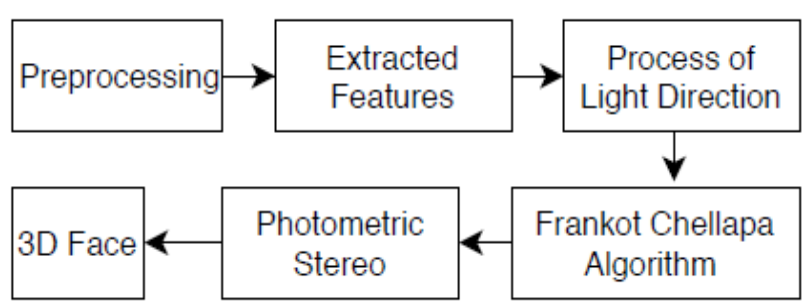

Fig. 1. The Overview of the Proposed System.

\section{Proprocessing of Input Images}

To perform preprocessing on input image can be used for the quality improvement of an image for further processing. In this paper, preprocessing contains the many steps of our proposed algorithm. The whole process of preprocessing is shown in Fig. 2. To start the preprocessing, first of all twodimensional image from dataset loaded to the proposed system and detect the desired face. After that it selects the rectangular region for the eye and forwarded to the step for the selection of the point. Next step it is converted into the mask as a result of the selection points from previous steps. Afterward it divided the image into left iris and right iris. Finally after extracting the eye portion from the image it is ready for the computation of the eyes features moves to the features eye center, eye radius and highlighted features are the parameters connects to the algorithm to process further steps as a whole to produce desired three-dimensional face reconstruction.

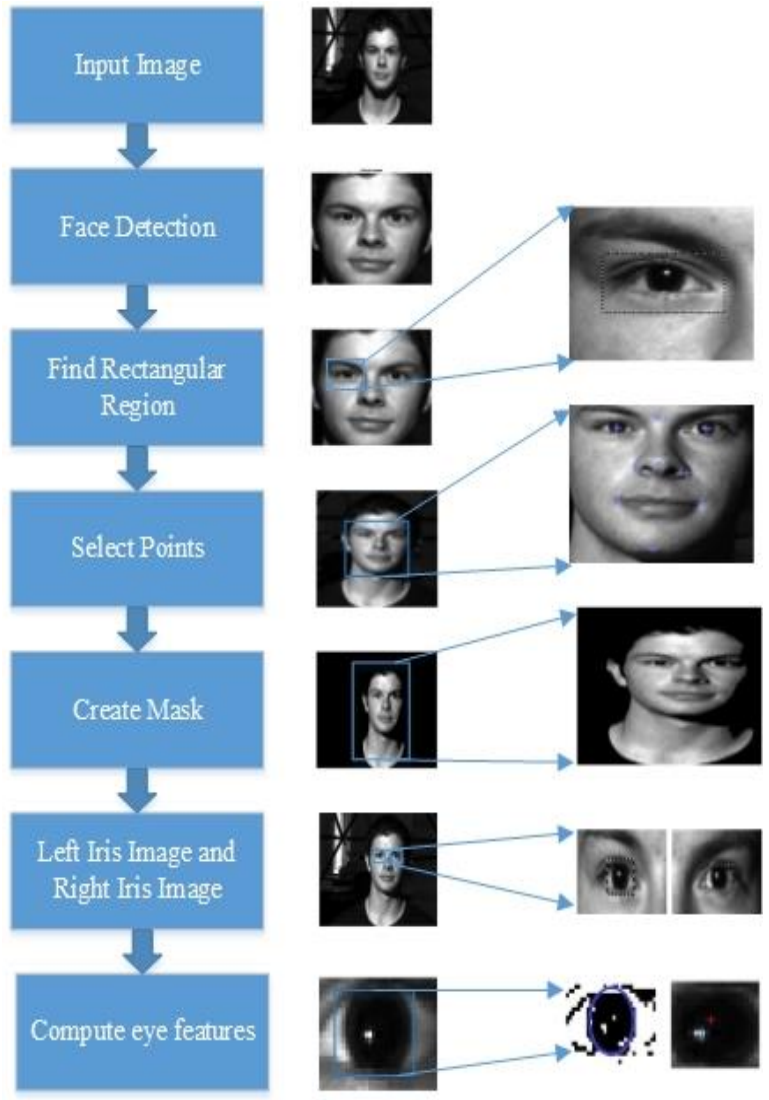

Fig. 2. The Overview of Preprocessing Steps of the Proposed System.

\section{Compute Light Direction}

We will take the single image under different light direction with different angles. Light reflects on eye. It only detects that eye that is taken from 90 angles. Fig. 3 shows light directions from eyes'. $\mathrm{x}$ is the angle of incidence, $\mathrm{y}$ is the angle of reflection and $\mathrm{P}$ is the normal that is to be computed. Use the Eyes' bright spot (Reflective Highlight). Angle of incidence is equal to angle of reflection for specular reflections where $\mathrm{x}=\mathrm{y}$. Direction of light from the source towards the eye. Direction of reflected light from the eye towards the camera to compute the normal we need the geometry of eye.

To find the normal we required the following information. It's important to compute the iris circle radius and the cornea sphere radius. Anatomically for adults: Iris circle radius = $12 \mathrm{~mm}$. Cornea sphere radius $=8 \mathrm{~mm}$. These values hardly differ in adults. The Normal at a certain point can be computed with the above information. Surface normal $\mathrm{P}$ is a vector whose value is needed to be figure out. It can be done through cross vector products. If surface is $\mathrm{P}$ is implicit as the set of points $(\mathrm{m}, \mathrm{n}, \mathrm{t})$ satisfying $\mathrm{F}(\mathrm{m}, \mathrm{n}, \mathrm{t})$, then normal at a point $(\mathrm{m}, \mathrm{n}, \mathrm{t})$ on the surface is given by the gradient $F(m, n, t) \nabla$. The gradient at any point is perpendicular to the level set. Whereas $\mathrm{F}(\mathrm{m}, \mathrm{n}, \mathrm{t})=0$ is the level set of the F. The implicit form of gradient can be also writes as:

$\mathrm{F}(\mathrm{m}, \mathrm{n}, \mathrm{t})=\mathrm{F}(\mathrm{m}, \mathrm{n})-\mathrm{t}$

These two forms relate to the analysis of surface being focused on upwards or downwards directions respectively. Frankot-Chellappa algorithm reconstructs the surface $\mathrm{P}$ by projecting $\{u, v\}$ on the set of integral Fourier functions. The Euler equation provides equation (3).

$\partial \mathrm{E} / \partial \mathrm{P}=\operatorname{div}(\partial \mathrm{E} / \partial \mathrm{Pm}, \partial \mathrm{E} / \partial \mathrm{Pn})$

Consider four functions $\mathrm{f} 1, \mathrm{f} 2, \mathrm{f} 3$ and $\mathrm{f} 4$ in equation 6 having following values:

$\partial \mathrm{E} / \partial \mathrm{Pm}=\mathrm{f} 1(\mathrm{Pm}, \mathrm{Pn})-\mathrm{f3}(\mathrm{u}, \mathrm{v}), \partial \mathrm{E} / \partial \mathrm{Pn}=\mathrm{f} 2(\mathrm{Pm}, \mathrm{Pn})-\mathrm{f} 4(\mathrm{u}$, v)

Putting the equation (6) in (7) we get $\operatorname{div}(\mathrm{f} 1(\mathrm{Pm}, \mathrm{Pn}), \mathrm{f} 2(\mathrm{Pm}, \mathrm{Pn}))-\partial \mathrm{E} / \partial \mathrm{P}=\operatorname{div}(\mathrm{f} 3(\mathrm{u}, \mathrm{v}), \mathrm{f} 4(\mathrm{u}$, v)) 
Above equations showed the previous working or the general framework. The extension of the Frankot-Chellappa algorithm is following

$\omega(\mathrm{m}, \mathrm{n}, \grave{\varepsilon} \mathrm{m}, \grave{\varepsilon ̀ n})=$ ei $(\grave{\varepsilon} \mathrm{m} \mathrm{m}, \grave{\varepsilon} n \mathrm{n})$,

We have $\oplus \mathrm{m}=\mathrm{i}$ غ̀m $\omega, \emptyset n=i$ غ̀n $\omega$. $\{\grave{\varepsilon} m, \grave{\varepsilon ̀ n}\}$ denote the improvement gradient field.

By substituting

$\partial \mathrm{E} / \partial \mathrm{P}=0, \mathrm{f} 1(\mathrm{Pm}, \mathrm{Pn})=\mathrm{F}(\mathrm{Pm}) \boldsymbol{\varphi}, \mathrm{f} 2(\mathrm{Pm}, \mathrm{Pn})=\mathrm{F}(\mathrm{Pn}) \boldsymbol{\varphi}, \mathrm{f}$ $(\mathrm{u}, \mathrm{v})=\mathrm{F}(\mathrm{u}) \oplus, \mathrm{f} 4(\mathrm{u}, \mathrm{v})=\mathrm{F}(\mathrm{v}) \oplus$ in equation $(7)$ we get

$\operatorname{div}(\mathrm{F}(\mathrm{Pm}) \varphi, F(\mathrm{Pn}) \varphi)=\operatorname{div}(\mathrm{F}(\mathrm{u}) \varphi, F(\mathrm{v}) \varphi)$,

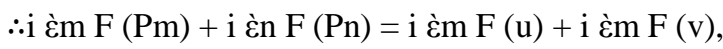

$\therefore-(\grave{\varepsilon} m 2+\grave{\varepsilon} n 2) F(P)=i(\grave{\varepsilon m ~ F ~}(\mathrm{u})+\grave{\varepsilon} m \mathrm{~F}(\mathrm{v}))$

$\mathrm{F}(\mathrm{P})=(-\mathrm{i}(\grave{\varepsilon} \mathrm{m} F(\mathrm{u})+\grave{\varepsilon} \mathrm{m} F(\mathrm{v}) /(\grave{\varepsilon} \mathrm{m} 2+\grave{\varepsilon} n 2))$

$\mathrm{P}=\mathrm{F}-1(-\mathrm{i}(\grave{\varepsilon} \mathrm{m} \mathrm{F}(\mathrm{u})+\grave{\varepsilon} \mathrm{m} \mathrm{F}(\mathrm{v}) /(\grave{\varepsilon} \mathrm{m} 2+\grave{\varepsilon ̀ n} 2))$

Equation (8) present reconstructs the 3D model. Whereas, $\mathrm{P}$ is the surface normal that has to be computed, $\mathrm{i}$ is the error function, $\mathrm{E}$ is the differentiable function, and $\omega$ is the orthonormal function.

\section{EXPERIMENTAL RESULTS}

The proposed system evaluated on Dataset of Sivam. The results of proposed method have been shown in Fig. 4 and Fig. 5. It depict that the proposed system results are more improved and fined than the previous FRMS algorithm, it has the issue with faces edges and quality of image, and the preprocessing was not very accurate. The proposed algorithm not only covers such issues but it also more robust and fast as compare to the previous approach more efficient and showed better results.

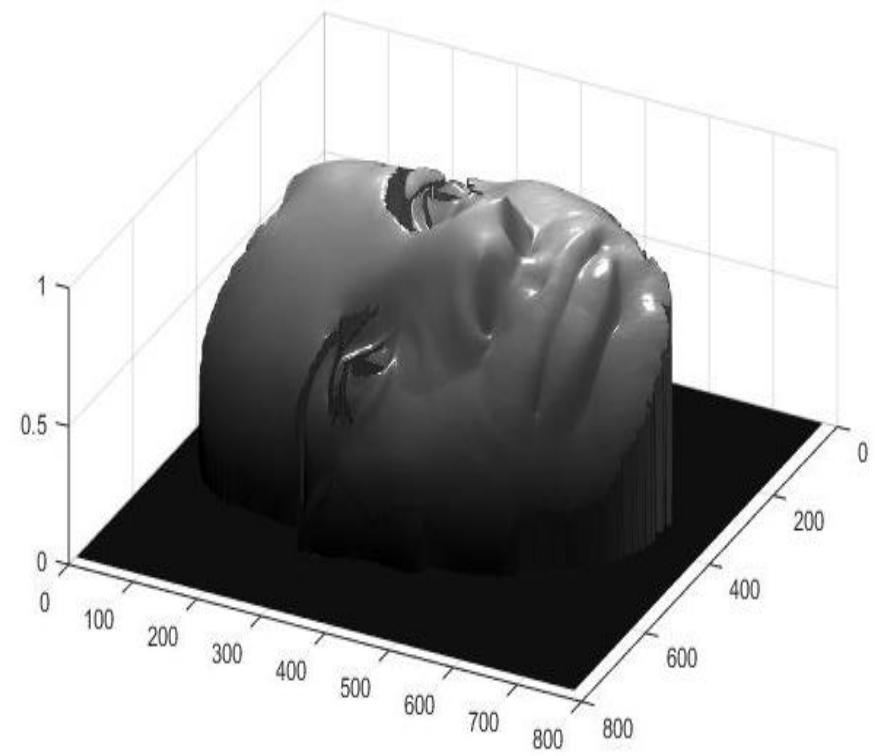

Fig. 4. Proposed System Output (Dataset Courtesy of Sivam Data Set).

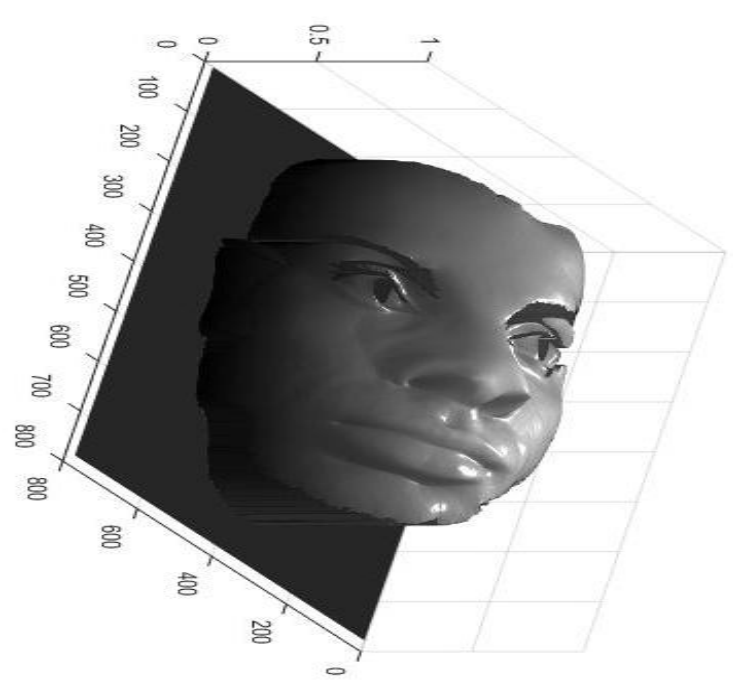

Fig. 5. Proposed System Output (Dataset Courtesy of Sivam Data Set).

A. Comparison with Existing Method

We compare our proposed method with our previous FRMS approach [5] is shown in Table 1.

TABLE I. COMPARISON WITH EXISTING METHOD

\begin{tabular}{|l|l|l|}
\hline Parameters & $\begin{array}{l}\text { Approach } \\
\text { Previous FRMS } \\
\text { approach }\end{array}$ & $\begin{array}{l}\text { Proposed } \\
\text { Technique }\end{array}$ \\
\hline Automatic Face Detection & No & Yes \\
\hline Fourier Transform & No & Yes \\
\hline Execution Time & $79-88 \mathrm{Sec}$ & $70-85 \mathrm{Sec}$ \\
\hline Output & Fig. 6 & Fig. 4 and Fig. 5 \\
\hline
\end{tabular}

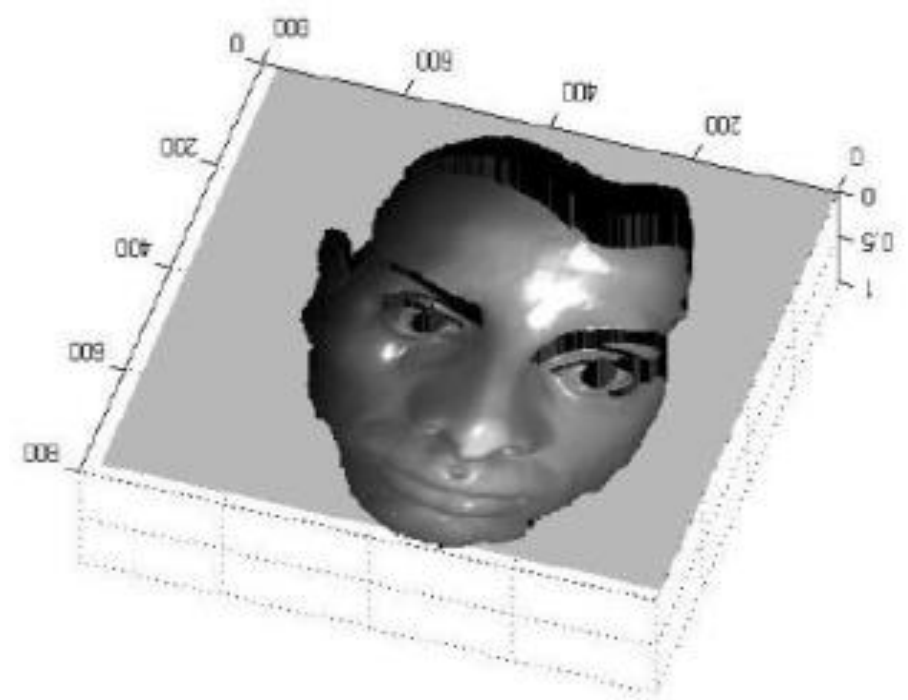

Fig. 6. Output of FRMS Approach [5]. 


\section{CONCLUSION}

In this paper we extend the FRMS approach [5] of the three dimensional methods in more detail, and also present the proposed technique exist in the category of photometric stereos. Reconstruct the 3D face from multiple images of different lighting condition. Proposed method provide the automatic detection of face and using face feature eyes to do segmentation of iris along with statistical features of pupil location. It works on minimum selection of six points choosing from face features and where iris as a result generates $3 \mathrm{D}$ face.

\section{FUTURE WORK}

The proposed algorithm can be further extend by using other features of face, the result can be more improved by converting into RGB image, applying color texture, this algorithm can be applicable on facial expression images to convert into three face, moreover the morphable model can also use for more better result of input images, And for the validation of algorithm can be applied on other dataset.

\section{ACKNOWLEDGMENT}

Author is thankful to all who contribute and support this project to make it successful.

\section{REFERENCES}

[1] Khan, M. S., Jehanzeb, M., Babar, M. I., Faisal, S., Ullah, Z., \& Amin, S. Z. B. M. (2018, August). Face Recognition Analysis Using 3D Model. In International Conference for Emerging Technologies in Computing (pp. 220-236). Springer, Cham.

[2] Richardson, E., Sela, M., \& Kimmel, R. (2016, October). 3D face reconstruction by learning from synthetic data. In 3D Vision (3DV), 2016 Fourth International Conference on (pp. 460-469). IEEE.
[3] Sela, M., Richardson, E., \& Kimmel, R. (2017, October). Unrestricted facial geometry reconstruction using image-to-image translation. In Computer Vision (ICCV), 2017 IEEE International Conference on (pp. 1585-1594). IEEE.

[4] Hassner, T., Harel, S., Paz, E., \& Enbar, R. (2015). Effective face frontalization in unconstrained images. In Proceedings of the IEEE Conference on Computer Vision and Pattern Recognition (pp. 42954304).

[5] Khan, M. S., \& Ullah, Z. (2017, February). A Proposed (FRMS) 3D Face Reconstruction Method from Stereo Images. In Proceedings of the 9th International Conference on Computer and Automation Engineering (pp. 150-154). ACM.

[6] Shi, B., Wu, Z., Mo, Z., Duan, D., Yeung, S. K., \& Tan, P. (2016). A benchmark dataset and evaluation for non-lambertian and uncalibrated photometric stereo. In Proceedings of the IEEE Conference on Computer Vision and Pattern Recognition (pp. 3707-3716).

[7] Santo, H., Samejima, M., Sugano, Y., Shi, B., \& Matsushita, Y. (2017, October). Deep photometric stereo network. In Proceedings of the IEEE International Conference on Computer Vision (pp. 501-509).

[8] QUeau, Y., Wu, T., Lauze, F., Durou, J. D., \& Cremers, D. (2017, July). A non-convex variational approach to photometric stereo under inaccurate lighting. In Proceedings of the 30th IEEE Conference on Computer Vision and Pattern Recognition. Honolulu, USA (Vol. 3, pp. 14-15).

[9] Logothetis, F., Mecca, R., \& Cipolla, R. (2017, July). Semi-calibrated near field photometric stereo. In Proceedings of the IEEE Conference on Computer Vision and Pattern Recognition. Honolulu, USA (Vol. 3, No. 5, p. 8).

[10] J. Roth, Y. Tong, \& X. Liu. Unconstrained 3D face reconstruction. In: 2015 IEEE Conference on Computer Vision and Pattern Recognition (CVPR).

[11] Sun, Y., Dong, J., Jian, M., \& Qi, L. (2015). Fast 3D face reconstruction based on uncalibrated photometric stereo. Multimedia Tools and Applications, 74(11), 3635-3650.

[12] Ullah, Zabeeh, Imran Mumtaz, and Muhammad Sajid Khan. "Analysis of 3D face modeling." International Journal of Signal Processing, Image Processing and Pattern Recognition 8.11 (2015): 7-14. 